Document downloaded from:

http://hdl.handle.net/10251/144104

This paper must be cited as:

Salas-Molina, F.; Rodriguez-Aguilar, JA.; Pla Santamaría, D. (31-0). Boundless multiobjective models for cash management. Engineering Economist (Online). 63(4):363381. https://doi.org/10.1080/0013791X.2018.1456596

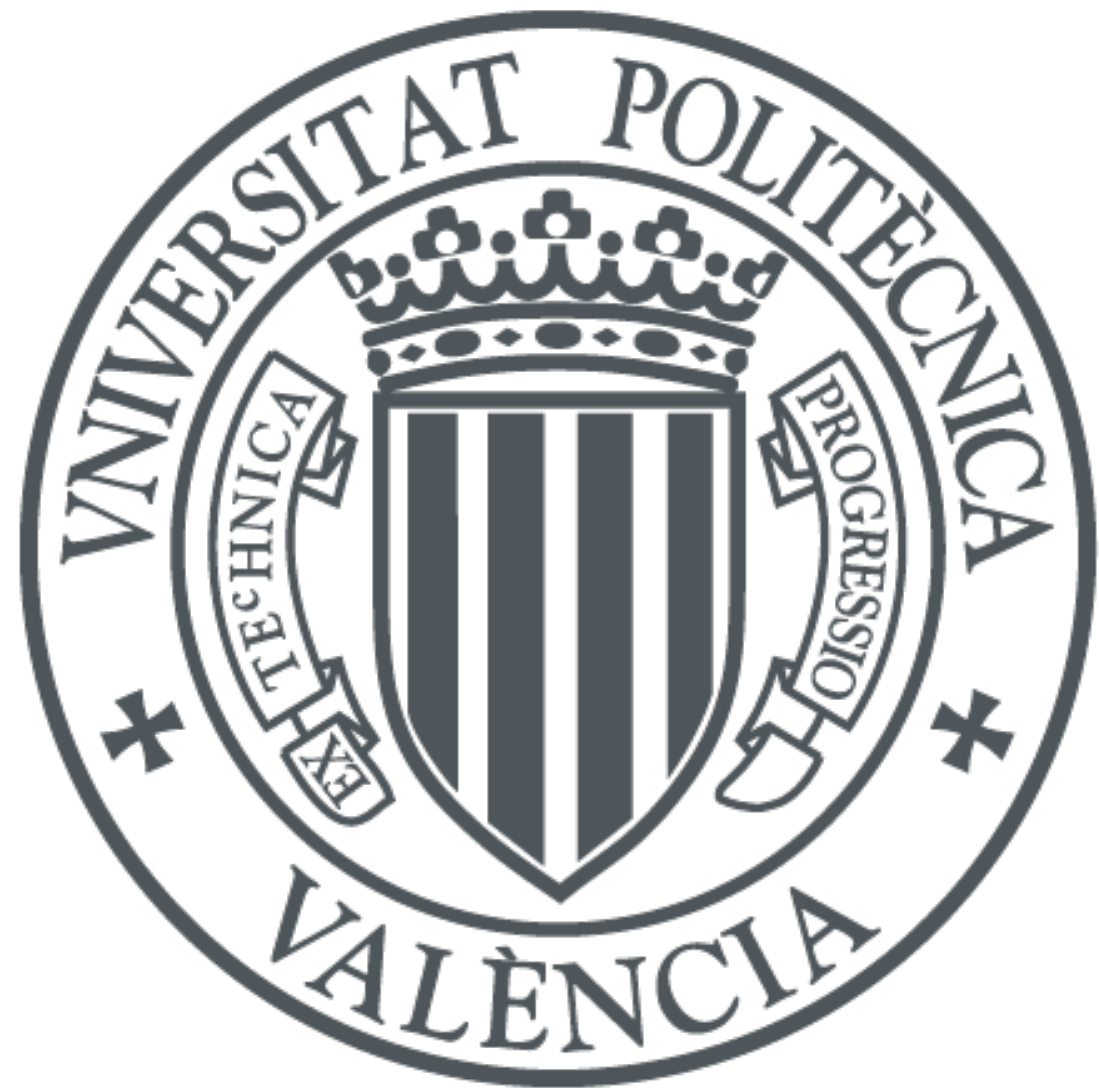

The final publication is available at

https://doi.org/10.1080/0013791X.2018.1456596

Copyright Taylor \& Francis

Additional Information

"This is an Accepted Manuscript of an article published by Taylor \& Francis in Engineering Economist on 31-05-2018, available online:

https://doi.org/10.1080/0013791X.2018.1456596" 


\title{
Boundless multiobjective models for cash management
}

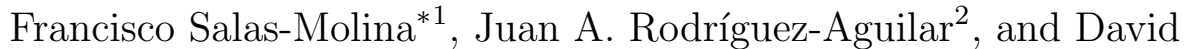 \\ Pla-Santamaria ${ }^{3}$ \\ ${ }^{1}$ Hilaturas Ferre, S.A., Les Molines, 2, 03450 Banyeres de Mariola, Spain \\ ${ }^{2}$ IIIA-CSIC, Campus UAB, 08913 Cerdanyola, Spain \\ ${ }^{3}$ Universitat Politècnica de València, Ferrándiz y Carbonell, 03801 Alcoy, Spain
}

June 3, 2018

\begin{abstract}
Cash management models are usually based on a set of bounds that complicates the selection of the optimal policies due to non-linearity. We here propose to linearize cash management models to guarantee optimality through linear-quadratic multiobjective compromise programming models. We illustrate our approach through a reformulation of the suboptimal state-of-the-art Gormley-Meade's model to achieve optimality. Furthermore, we introduce a much simpler formulation that we call the Boundless Model that also provides optimal solutions without using bounds. Results from a sensitivity analysis using real data sets from 54 different companies show that our Boundless Model is highly robust to cash flow prediction errors.
\end{abstract}

Keywords Working capital; forecasting; linear-quadratic programming; risk.

\section{Introduction}

Cash managers have to make daily decisions about the amount of transactions between cash holdings and any other kind of available investment asset. On the one hand, a certain amount of cash must be kept for operational and precautionary purposes. On the other hand, idle cash balances may be invested in short-term assets such as interest-bearing accounts or treasury bills for a profit. As a result, working capital management has deserved the attention of the research community for decades (see e.g. Miller and Orr (1966); Keown and Martin (1977); Xu and Birge (2008)).

The ultimate goal of the cash management problem (CMP) is to find the best sequence of control actions over a given planning horizon which is called a

\footnotetext{
*Corresponding author. E-mail addresses: francisco.salas.molina@gmail.com, jar@iiia.csic.es,dplasan@upv.es
} 
policy. The typical framework in the CMP considers two assets, namely, a cash account and an investment account where idle cash balances are allocated for a profit. In addition, most cash management models have a common feature: they are based on setting control limits or bounds. In other words, they can be considered as Bound-Based Models (BBM). In a BBM, the sequence of control actions is attained through a set of bounds determining the form of the policy. Cash balance is allowed to wander around between some bounds, usually a high bound and a low bound. When any of these bounds is reached, a control action is made to restore the balance to some target level.

Since Miller and Orr (1966), different models have been proposed to address the CMP by means of bounds as surveyed in Gregory (1976); Srinivasan and Kim (1986); da Costa Moraes et al. (2015). However, the method to elicit a particular set of bounds and its motivation varies with the model. For instance, the BBM by Miller and Orr (1966) is based on three bounds and Girgis (1968); Penttinen (1991) and Gormley and Meade (2007) considered four bounds to account for both fixed and linear transaction costs. Stone (1972) proposed the use of forecasts and five bounds, Neave (1970) and later Chen and SimchiLevi (2009) proposed optimal policies based on six bounds. Cash management models are usually linked to the assumption of a particular cash flow process ranging from deterministic cash flows in Baumol (1952), to purely stochastic behavior in Miller and Orr (1966); Constantinides and Richard (1978); Bar-Ilan et al. (2004); Baccarin (2009), which usually implies a normal, independent and stationary cash flow distribution.

However, non-linearity introduced by bounds complicates the selection of optimal policies. For instance, Gormley and Meade (2007) and da Costa Moraes and Nagano (2014) suggested the use of evolutionary algorithms to solve the CMP. However, this approach does not guarantee the optimality of the cash management solutions. In order to overcome this limitation, we here propose a reformulation of the CMP through mathematical programming.

In addition, risk management is an ongoing issue in finance (Artzner et al., 1999; Rockafellar and Uryasev, 2002; Park and Herath, 2000). Indeed, cash managers may be interested not only in the cost of alternative policies but also in the risk (Salas-Molina et al., 2016). In order to include risk as an additional goal, we encode the CMP as a linear-quadratic multiple-criteria decision-making model (Yu, 1985; Ballestero and Romero, 1998). We illustrate our approach through a multiobjective reformulation of the Gormley and Meade (2007) model (MOGM).

In this paper, we introduce a novel formulation, the so-called Boundless Model (BM), which returns the best sequence of control actions without using bounds. This BM does not impose restrictions on the form of possible policies and uses forecasts as a key input. We demonstrate that our BM is a much simpler CMP formulation that guarantees the optimality of the solutions against approximate solutions of a state-of-the-art BBM. Furthermore, we empirically show that our BM outperforms the MOGM in terms of required run time to solve the CMP.

A critical issue when dealing with cash management models using forecasts as a key input is the influence of forecasting errors on the performance of the model. In order to evaluate this impact, we present a sensitivity analysis of our BM to forecasting errors using 54 real data sets from different companies. The results show that our BM is highly robust to prediction errors. A further 
advantage of this method is that it allows to estimate the reward that can be obtained by improving predictive accuracy.

Summarizing, the results presented in this paper support decision-making in cash management by considering cash flow forecasts and multiobjective programming. In addition, we solve important open research questions in cash management through the following four main contributions:

1. A linear formulation of the CMP to guarantee the optimality of solutions.

2. A linear-quadratic multiobjective reformulation of the Gormley-Meade's model that, unlike the original model, guarantees the optimality of solutions.

3. A novel cash management model, namely, the BM, that provides optimal solutions and generalizes several state-of-the-art models.

4. A sensitivity analysis of the BM to forecasting errors.

In what follows, we provide useful background on the multiobjective cash management problem and the Gormley and Meade (2007) model in Section 2. In Section 3, we linearize the cash management problem that in Section 4 we apply to reformulate the Gormley and Meade (2007) model. Next, in Section 5, we introduce the BM for multiobjective cash management. Section 6 analyzes the impact of forecasting errors in the BM's performance. Finally, Section 7 provides some concluding remarks.

\section{Background}

The CMP has been usually approached as a control problem. The cash balance of any bank account needs to be monitored to keep the balance between two bounds, for instance, a low bound and a high bound. To this end, some control actions are taken to increase or decrease the balance according to some rules in order to minimize expected holding and transaction costs. The set of control actions deployed over a planning horizon, namely, the policy, is the solution to the CMP. However, cash managers may be interested in the cost but also in the risk of alternative policies. In order to analyze both cost and risk, in this section, we first formulate the CMP as a multiobjective program as proposed in SalasMolina et al. (2016). Next, we provide useful background on the state-of-the-art Gormley and Meade (2007) cash management model.

\subsection{Formulation of the multiobjective cash management problem}

We here introduce the multiobjective cash management problem (MOCMP) as proposed by Salas-Molina et al. (2016). The MOCMP is defined as an optimization problem whose goal is to find the best sequence of transactions $X=\left\langle x_{1}, \ldots, x_{t}, \ldots, x_{T}\right\rangle$, with $x_{t} \in \mathbb{R}, t \in \mathbb{N}$, and $1 \leq t \leq T$ for a given planning horizon $T \in \mathbb{N}$. The solution to the MOCMP is the policy that optimizes some objective function considering both cost and risk, but maybe other interesting goals. The cash balance at the end of the day is computed as the sum 
of the initial cash balance $b_{t_{1}}$, the actual cash flow $f_{t}$ and the control action $x_{t}$, according to the following state transition law:

$$
b_{t}=b_{t-1}+f_{t}+x_{t}
$$

Within the framework described in equation (1), a positive transaction $x_{t}>0$, adding into a cash account by selling available investments, is called an ordering transaction. Similarly, a negative transaction $x_{t}<0$, withdrawing from a cash account by buying additional investments, is called a returning transaction. The MOCMP is characterized by its particular cost structure. More precisely, any ordering transaction may have a cost, which may include a fixed part $\left(\gamma_{0}^{+}\right)$and a variable part $\left(\gamma_{1}^{+}\right)$. On the other hand, a return transaction from a cash account may also have a cost with a fixed part $\left(\gamma_{0}^{-}\right)$and a variable part $\left(\gamma_{1}^{-}\right)$. Furthermore, at the end of the day, a holding cost $(h)$ per money unit is charged if a positive cash balance occurs, or a penalty cost $(u)$ per money unit is charged if a negative cash balance occurs. According to this cost structure, a general daily cost function is defined as:

$$
c\left(x_{t}\right)=\Gamma\left(x_{t}\right)+H\left(b_{t}\right)
$$

where $x_{t}$ is the transaction made at day $t, b_{t}$ is the cash balance at the end of day $t, \Gamma\left(x_{t}\right)$ is a transfer cost function, and $H\left(b_{t}\right)$ stands for a holding/shortage cost function. The transfer cost function $\Gamma\left(x_{t}\right)$ is defined as:

$$
\Gamma\left(x_{t}\right)=\left\{\begin{array}{lll}
\gamma_{0}^{-}-\gamma_{1}^{-} \cdot x_{t} & \text { if } & x_{t}<0 \\
0 & \text { if } & x_{t}=0, \\
\gamma_{0}^{+}+\gamma_{1}^{+} \cdot x_{t} & \text { if } & x_{t}>0
\end{array}\right.
$$

Additionally, the holding/shortage cost function is expressed as:

$$
H\left(b_{t}\right)= \begin{cases}-u \cdot b_{t} & \text { if } \quad b_{t}<0 ; u \geq 0 \\ h \cdot b_{t} & \text { if } \quad b_{t} \geq 0 ; h \geq 0\end{cases}
$$

Under this cost structure, the ultimate goal of the MOCMP defined in SalasMolina et al. (2016) is to find the policy $X$ that minimizes the expected cost and risk over the time horizon $T$. To this end, the expected cost $C(X)$ is measured by the average daily cost:

$$
C(X)=\frac{1}{T} \sum_{t=1}^{T} c\left(x_{t}\right)=\frac{1}{T} \sum_{t=1}^{T}\left[\Gamma\left(x_{t}\right)+H\left(b_{t}\right)\right]
$$

and the expected risk $R(X)$ is measured by the standard deviation of the daily cost:

$$
R(X)=\left(\frac{1}{T} \sum_{t=1}^{T}\left(C(X)-c\left(x_{t}\right)\right)^{2}\right)^{1 / 2} .
$$

Then, under the framework of compromise programming (Ballestero and Romero, 1998), the goal is to find policy $X$ that minimizes a loss function $\mathcal{L}_{\alpha}(X)$ computing weighted distances to an ideal point where both cost and risk are zero:

$$
\min \mathcal{L}_{\alpha}(X)=\min \left[\left(\frac{w_{1}}{C_{\max }} \cdot C(X)\right)^{\alpha}+\left(\frac{w_{2}}{R_{\max }} \cdot R(X)\right)^{\alpha}\right]^{1 / \alpha}
$$


subject to:

$$
X \in S
$$

where $w_{1}$ and $w_{2}$ are weights defining the particular cost/risk preferences of cash managers, $\alpha$ is a positive integer, and $S$ is the set of all possible policies. In what follows, we consider only Manhattan distances to the ideal point by setting $\alpha=1$ in objective function (7) to guarantee linearity. Furthermore, $C_{\max }$ and $R_{\max }$ can be regarded either as budget limitations or as normalization factors for comparative purposes. Next, we provide background on a BBM that defines $S$ as a dynamic simple policy using forecasts as a key input to the model.

\subsection{The Gormley-Meade (GM) cash management model}

Cash managers can leverage cash flow forecasts to reduce the uncertainty within a short-term planning horizon (Stone, 1972; Stone and Miller, 1987; SalasMolina et al., 2017). To this end, Gormley and Meade (2007) proposed a cash management model based on forecasts and four bounds: a low bound $D$, a high bound $V$ and two target levels $v$ and $d$. The cash balance is allowed to wander around between these bounds as shown in Figure 1.

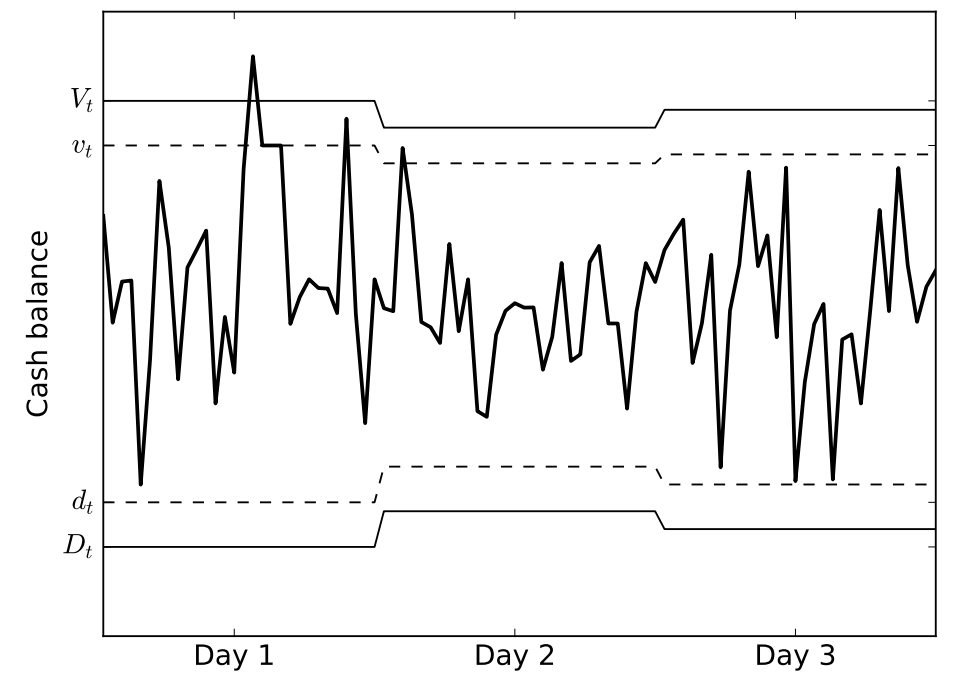

Figure 1: The dynamic simple policy of Gormley-Meade.

When $V$ is reached a withdrawal transfer is made to restore the balance to a target level $v$. In the same way, when the cash balance reaches $D$, a positive transfer is made to restore the balance to a target $d$. Forecasts for a given planning horizon are used as a key input to the model that establishes a dynamic simple policy of the form:

$$
x_{t}=\left\{\begin{array}{lll}
v_{t}-\hat{b}_{t-1}-\hat{f}_{t}, & \text { if } & \hat{b}_{t-1}+\hat{f}_{t}>V_{t}, \\
0, & & \text { otherwise } \\
d_{t}-\hat{b}_{t-1}-\hat{f}_{t}, & \text { if } & \hat{b}_{t-1}+\hat{f}_{t}<D_{t}
\end{array}\right.
$$


where $D_{t}, d_{t}, v_{t}, V_{t}$, for $1 \leq t \leq T$ are the decision variables satisfying $D_{t} \leq$ $d_{t} \leq v_{t} \leq V_{t}$, and $\hat{b}_{t}$ and $\hat{f}_{t}$ are the predicted cash balance and cash flow at time $t$, respectively. This model is dynamic and the decision variables $D_{t}, d_{t}, v_{t}, V_{t}$ must be obtained at each time step $t$. However, due to the non-linearity of the form of the policy described in (9), the authors proposed the use evolutionary algorithms to solve the CMP without guaranteeing the optimality of solutions. In Section 4, we present a reformulation of this model that overcomes this limitation.

\section{Linearizing the cash management problem}

In this section, we formulate the CMP as a linear program since we aim to guarantee the optimality of solutions. In order to linearize the common two-assets setting of the CMP, consider a company with two bank accounts as depicted in Figure 2. Account 1 receives payments from customers (inflows) and it is also used to send payments to suppliers (outflows). Both inflows and outflows are summarized through the net cash flow $f_{t}$.

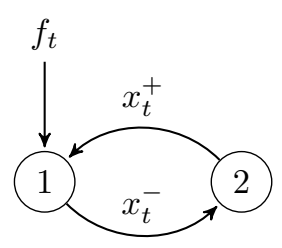

Figure 2: The common two-assets setting in the cash management problem.

Let $x_{t}$ be the difference between positive and negative control actions $x_{t}=$ $x_{t}^{+}-x_{t}^{-}$at bank account 1 with $x_{t}^{+}$and $x_{t}^{-}$being non-negative real numbers. Then, account 2 represents the amount of alternative investments available to be converted into cash through transaction $x_{t}^{+}$when needed. In addition, idle cash balances from account 1 can be allocated in account 2 for a profit through transaction $x_{t}^{-}$. In this setting, the transfer cost function in equation (3) can be expressed as follows:

$$
\Gamma\left(x_{t}\right)=z_{t}^{+} \cdot \gamma_{0}^{+}+\gamma_{1}^{+} \cdot x_{t}^{+}+z_{t}^{-} \cdot \gamma_{0}^{-}+\gamma_{1}^{-} \cdot x_{t}^{-}
$$

where $z_{t}^{+}, z_{t}^{-} \in\{0,1\}$ are binary auxiliary variables satisfying:

$$
\begin{gathered}
z_{t}^{+}+z_{t}^{-} \leq 1 \\
m \cdot z_{t}^{+} \leq x_{t}^{+} \leq M \cdot z_{t}^{+} \\
m \cdot z_{t}^{-} \leq x_{t}^{-} \leq M \cdot z_{t}^{-}
\end{gathered}
$$

where $M(m)$ is a very large (small) number. Note that although we restrict $x_{t}^{+}$ and $x_{t}^{-}$to be non-negative numbers, the left-hand side of equations (12) and (13) are necessary to ensure $z_{t}^{+}, z_{t}^{-}=0 \Leftrightarrow x_{t}^{+}, x_{t}^{-}=0$. A similar approach can be followed to linearize the holding/penalty cost function (4) through:

$$
H\left(b_{t}\right)=z_{t} \cdot h \cdot b_{t}-\left(1-z_{t}\right) \cdot u \cdot b_{t}
$$


where $z_{t} \in\{0,1\}$ is another auxiliary binary variable satisfying:

$$
-M\left(1-z_{t}\right) \leq b_{t} \leq M \cdot z_{t} .
$$

However, since cash managers usually discard policies with negative balances due to high penalty costs, in what follows, we assume $u=\infty$ and $H\left(b_{t}\right)=h \cdot b_{t}$, which results in the following daily cost function equivalent to equation (2):

$$
c\left(x_{t}\right)=\gamma_{0}^{+} \cdot z_{t}^{+}+\gamma_{1}^{+} \cdot x_{t}^{+}+z_{t}^{-} \cdot \gamma_{0}^{-}+\gamma_{1}^{-} \cdot x_{t}^{-}+h \cdot b_{t} .
$$

Since control actions are taken in advance to real cash flow, we use predicted cash flows $\hat{f}_{t}$ and balances $\hat{b}_{t}$ instead of actual values in the state transition law in equation (1) as follows:

$$
\hat{b}_{t}=\hat{b}_{t-1}+\hat{f}_{t}+x_{t}^{+}-x_{t}^{-} .
$$

Next, we rely on the previous linear functions to derive optimal solutions from a reformulation of the GM model.

\section{Reformulating the Gormley-Meade model as a linear-quadratic program}

We mentioned in Section 2.2 that the form of the policy described in (9) within the GM model, implies facing a non-linear problem whose solution is by no means straightforward. The authors suggested the use of evolutionary algorithms to obtain the set of bounds $\left\{D_{t}, d_{t}, v_{t}, V_{t}\right\}$ for each time step $t$, but this method does not guarantee the optimality of the solutions. Evolutionary algorithms are iterative procedures based on direct search (Branke et al., 2008). A population of feasible solutions is randomly generated and better solutions are found by comparing the fitness of the new population to the current population. As a result, there is no way to test if a given solution is optimal. Unlike evolutionary algorithms, classical gradient-based optimization procedures such as linear programming allow to test the optimality of solutions (Nocedal and Wright, 2006; Bazaraa et al., 2011). Thus, we next present a linear-quadratic reformulation of the GM model allowing cash managers to obtain optimal solutions through:

- A linear program by considering only cost as a linear expression.

- A linear-quadratic program by considering a linear cost expression and a quadratic risk expression.

A third option may be considered by solving a linear program with both cost and risk linear expressions, e.g., by defining a linear measure of risk. However, since we are interested in a cost-risk optimization as described in Salas-Molina et al. (2016), we next formalize the second option. Then, within the framework of the GM model, positive transactions $x_{t}^{+}$occur when low bound $D_{t}$ is reached. Thus, $z_{t}^{+}=1$ when $\hat{b}_{t-1}+\hat{f}_{t} \leq D_{t}$ and the amount transferred is given by $x_{t}^{+}=d_{t}-\hat{b}_{t-1}-\hat{f}_{t}$. This can be expressed by the following linear constraints:

$$
\hat{b}_{t-1}+\hat{f}_{t}-D_{t} \leq M\left(1-z_{t}^{+}\right)
$$




$$
-M\left(1-z_{t}^{+}\right) \leq x_{t}^{+}-d_{t}+\hat{b}_{t-1}+\hat{f}_{t} \leq M\left(1-z_{t}^{+}\right) .
$$

Furthermore, negative transactions $x_{t}^{-}$occur when high bound $V_{t}$ is reached. Thus, $z_{t}^{-}=1$ when $\hat{b}_{t-1}+\hat{f}_{t} \geq V_{t}$ and the amount transferred is given by $x_{t}^{-}=\hat{b}_{t-1}+\hat{f}_{t}-v_{t}$. This can be expressed by the following linear constraints:

$$
\begin{gathered}
V_{t}-\hat{b}_{t-1}-\hat{f}_{t} \leq M\left(1-z_{t}^{-}\right) \\
-M\left(1-z_{t}^{-}\right) \leq x_{t}^{-}+v_{t}-\hat{b}_{t-1}-\hat{f}_{t} \leq M\left(1-z_{t}^{-}\right) .
\end{gathered}
$$

A third group of conditions must hold when the cash balance is between bounds $D_{t}$ and $V_{t}$. Thus, when $z_{t}^{+}=0$ and $z_{t}^{-}=0$ no transaction occurs. This can be expressed by the following linear constraints:

$$
\begin{gathered}
D_{t}-\hat{b}_{t-1}-\hat{f}_{t} \leq M\left(z_{t}^{+}+z_{t}^{-}\right) \\
\hat{b}_{t-1}+\hat{f}_{t}-V_{t} \leq M\left(z_{t}^{+}+z_{t}^{-}\right) \\
m \cdot z_{t}^{+} \leq x_{t}^{+} \leq M \cdot z_{t}^{+} \\
m \cdot z_{t}^{-} \leq x_{t}^{-} \leq M \cdot z_{t}^{-}
\end{gathered}
$$

As a result, we can reformulate the multiobjective cash management problem encoded in equations (7) and (8) to accommodate policies of the GM type as the following linear-quadratic multiobjective Gormley-Meade (MOGM) program:

$$
\min \left[\frac{w_{1}}{C_{\max }} C(X)+\frac{w_{2}}{R_{\max }} R(X)\right]
$$

subject to:

$$
\begin{gathered}
\hat{b}_{t}=\hat{b}_{t-1}+\hat{f}_{t}+x_{t}^{+}-x_{t}^{-} \\
c\left(x_{t}\right)=\gamma_{0}^{+} \cdot z_{t}^{+}+\gamma_{1}^{+} \cdot x_{t}^{+}+\gamma_{0}^{-} \cdot z_{t}^{-}+\gamma_{1}^{-} \cdot x_{t}^{-}+h \cdot b_{t} \\
M\left(z_{t}^{+}+z_{t}^{-}\right) \leq \hat{b}_{t-1}+\hat{f}_{t}-D_{t} \leq M\left(1-z_{t}^{+}\right) \\
-M\left(1-z_{t}^{+}\right) \leq x_{t}^{+}-d_{t}+\hat{b}_{t-1}+\hat{f}_{t} \leq M\left(1-z_{t}^{+}\right) . \\
M\left(z_{t}^{+}+z_{t}^{-}\right) \leq V_{t}-\hat{b}_{t-1}-\hat{f}_{t} \leq M\left(1-z_{t}^{-}\right) \\
-M\left(1-z_{t}^{-}\right) \leq x_{t}^{-}+v_{t}-\hat{b}_{t-1}-\hat{f}_{t} \leq M\left(1-z_{t}^{-}\right) \\
D_{t} \leq d_{t} \leq v_{t} \leq V_{t} \\
z_{t}^{+}+z_{t}^{-} \leq 1 \\
m \cdot z_{t}^{+} \leq x_{t}^{+} \leq M \cdot z_{t}^{+} \\
m \cdot z_{t}^{-} \leq x_{t}^{-} \leq M \cdot z_{t}^{-} \\
\hat{b}_{t} \geq b_{\min } \\
w_{1}+w_{2}=1 \\
1 \leq t \leq T, t \in \mathbb{N}, T \in \mathbb{N}
\end{gathered}
$$

where the decision variables are the bounds $\left\{D_{t}, d_{t}, v_{t}, V_{t}\right\}$ that ultimately determine control action $x_{t} \in X$ for each time step $t$, and $C(X)$ and $R(X)$ are linear-quadratic cost and risk functions such as the ones defined in equations 
(5) and (6) that depend on policy $X$. Note that $\hat{b}_{t}, x_{t}^{+}, x_{t}^{-}, z_{t}^{+}$and $z_{t}^{-}$are also decision variables determining policy $X$ as we show in the upcoming section.

Normalization factors $C_{\max }$ and $R_{\max }$ are used to avoid numerical bias to one of the goals but also for comparative purposes. In this paper, we propose to set $C_{\max }$ and $R_{\max }$ to the expected cost and risk of a trivial policy consisting in taking no control action.

Following the recommendations in Ben-Tal et al. (2009) for robust optimization, we set a minimum cash balance proportional to the uncertainty introduced by forecasting errors. To this end, we assume that the cumulative forecasting error distribution for the planning horizon $T$ is known and presents standard deviation $\sigma_{e}$. Then, we set $b_{\min }=\xi \cdot \sigma_{e}$, where $\xi$ is a positive parameter. Assuming Gaussian forecasting errors, typical values for $\xi$ are 2 or 3 leading, respectively, to an unfeasible program in only $95 \%$ or $99 \%$ of the realizations of the error distribution.

An important advantage of the previous linear-quadratic reformulation in comparison to the original GM model is that it can be implemented and optimally solved through the modeling framework provided by mathematical programming solvers such as CPLEX or Gurobi. However, $8 \times T$ decision variables and $23 \times T$ constraints are involved. Hence, one may wonder if a simpler formulation is possible. Next, we present a novel formulation of the CMP, which we call the Boundless Model (BM), which aims to solve this problem.

\section{Boundless multiobjective models for cash man- agement}

In this section, we introduce a novel boundless multiobjective model for cash management. First, we formally describe the model. We next highlight a crucial property of this model and we finally perform a numerical comparison exercise between the MOGM and the BM.

\subsection{Model formulation}

In the GM model described in Section 2.2, cash managers have to determine the set of bounds $\left\{D_{t}, d_{t}, v_{t}, V_{t}\right\}$ which minimizes expected transaction and holding costs for the whole planning horizon $T$. Indeed, the bounds are previously obtained to the deployment of the policy according to equation (9). However, we argue that the ultimate goal of the cash management problem is not to find the best set of bounds, but the best sequence of control actions. As a result, we here propose to change the focus from bounds to the actual policy to simplify computations. To this end, we next present a BM for multiobjective cash management:

$$
\min \left[\frac{w_{1}}{C_{\max }} C(X)+\frac{w_{2}}{R_{\max }} R(X)\right]
$$

subject to:

$$
\begin{gathered}
\hat{b}_{t}=\hat{b}_{t-1}+\hat{f}_{t}+x_{t}^{+}-x_{t}^{-} \\
c\left(x_{t}\right)=\gamma_{0}^{+} \cdot z_{t}^{+}+\gamma_{1}^{+} \cdot x_{t}^{+}+\gamma_{0}^{-} \cdot z_{t}^{-}+\gamma_{1}^{-} \cdot x_{t}^{-}+h \cdot b_{t} \\
z_{t}^{+}+z_{t}^{-} \leq 1
\end{gathered}
$$




$$
\begin{gathered}
m \cdot z_{t}^{+} \leq x_{t}^{+} \leq M \cdot z_{t}^{+} \\
m \cdot z_{t}^{-} \leq x_{t}^{-} \leq M \cdot z_{t}^{-} \\
\hat{b}_{t} \geq b_{\min } \\
w_{1}+w_{2}=1 \\
1 \leq t \leq T, t \in \mathbb{N}, T \in \mathbb{N}
\end{gathered}
$$

Note that the set of constraints from equation (41) to (48) are only necessary to linearize the CMP as described in Section 3 and to achieve a certain degree of protection against forecasting errors by means of $\hat{b}_{t} \geq b_{\text {min }}$.

By setting $C(X)$ to the average daily cost and $R(X)$ to the standard deviation, or tantamount, the variance of daily costs, we face a quadratic programming optimization problem. Using basic algebra, the BM encoded from equations (40) to (48) can be rewritten as a sum of linear and quadratic terms of the decision variables. This sum can finally be expressed in matrix form to accommodate the standard quadratic formulation (see e.g. Nocedal and Wright (2006)):

$$
\min \frac{1}{2} \boldsymbol{x}^{\prime} \cdot G \cdot \boldsymbol{x}+\boldsymbol{x}^{\prime} \cdot \boldsymbol{g}
$$

subject to:

$$
\begin{aligned}
& a_{i}^{\prime} \cdot \boldsymbol{x}=d_{i}, \quad i \in \mathcal{E} \\
& a_{i}^{\prime} \cdot \boldsymbol{x} \geq d_{i}, \quad i \in \mathcal{I}
\end{aligned}
$$

where $\boldsymbol{x} \in \mathbb{R}^{n}$ is a vector of decision variables, $G$ is a symmetric $n \times n$ matrix, $\mathcal{E}$ and $\mathcal{I}$ are finite sets of indexes, and $\boldsymbol{g}$ and $\left[a_{i}\right]$ are also vectors in $\mathbb{R}^{n}$.

By specializing the Karush-Kuhn-Tucker optimality conditions to quadratic problems (Nocedal and Wright, 2006), if $\boldsymbol{x}^{*}$ satisfies these conditions, then $\boldsymbol{x}^{*}$ is a global solution of the program (49)-(51), hence guaranteeing the optimality of solutions provided by our BM.

\subsection{The equivalence theorem}

The BM model is clearly a much simpler formulation than that of MOGM since only $4 \times T$ decision variables and $8 \times T$ constraints are involved. However, since both models aim to obtain the optimal policy, both formulations must return the same optimal results for the same inputs as we next demonstrate.

As a simple numerical example, consider a predicted cash balance $\hat{b}_{t-1}=10$ and a forecast $\hat{f}_{t}=1$. Let us assume that the optimal policy is $x_{t}^{+}=4$. Then, the optimal bound $d_{t}$ is necessarily 15 since $d_{t}=x_{t}^{+}+\hat{b}_{t-1}+\hat{f}_{t}=4+10+1=15$ from equation (9). Indeed, the next simplified optimization problem:

$$
\min _{x, d} g(x, d)
$$

subject to:

$$
x=d+a
$$

where $x$ and $d$ are the decision variables and $a$ is a constant, is equivalent to:

$$
\min _{x} g(x, x-a)=\min _{x} g(x)
$$

subject to no constraint. This observation leads us to formulate the following theorem. 
Theorem 1. Given a forecast $\hat{f}_{t}$ for $1 \leq t \leq T$, a minimum balance $b_{\text {min }}$, a cost structure $\beta=\left\{\gamma_{0}^{+}, \gamma_{0}^{-}, \gamma_{1}^{+}, \gamma_{1}^{-}, h\right\}$, two normalization factors $C_{\max }$ and $R_{\text {max }}$, and some weights $w_{1}$ and $w_{2}$, the BM encoded from equation (40) to (48) and the MOGM model encoded from equation (26) to (39) are equivalent in the sense that both models return the same optimal solution for the very same input.

Proof. Optimizing a set of bounds for each time step is equivalent to directly optimizing the policy since the former determines the latter and vice versa. Let us assume that $\hat{b}_{t-1}+\hat{f}_{t} \leq D_{t}$ holds. Then, finding $d_{t}$ that minimizes some objective function subject to $x_{t}^{+}=d_{t}-\hat{b}_{t-1}-\hat{f}_{t}$ from equation (9) is equivalent to finding $x_{t}^{+}$due to the direct relationship between $x_{t}^{+}$and $d_{t}$. A similar reasoning leads to the same conclusion when $\hat{b}_{t-1}+\hat{f}_{t} \geq V_{t}$ or when $D_{t} \leq \hat{b}_{t-1}+\hat{f}_{t} \leq V_{t}$. This fact implies that the set of constraints from (29) to (33) are redundant and can be removed from the optimization process demonstrating that both models are equivalent.

Furthermore, our BM provides a general framework for cash management in several aspects or dimensions of the problem. First, our BM accepts any type cash flow process from a deterministic one (Baumol, 1952) to a pure stochastic one (Miller and Orr, 1966) and also empirical data sets of cash flows (Gormley and Meade, 2007; Salas-Molina et al., 2016). Our BM simplifies the Gormley and Meade (2007) cash management model by removing half of the decision variables and almost two thirds of the constraints. In addition, when no forecast is used (Penttinen, 1991), forecasts can be set to zero. Our MOGM model is also a generalization of the Miller and Orr (1966) model that can be obtained by setting forecasts to zero, and by forcing that $v_{t}=d_{t}$ and $D_{t}, d_{t}, v_{t}$ and $V_{t}$ to be constant for the whole planning horizon.

\subsection{Comparing the MOGM, the BM and the GM}

In this section, we first provide an illustrative example comparing the solutions given by the GM model using genetic algorithms to those provided by the MOGM reformulation and the new BM using quadratic programming. The use of genetic algorithms in the GM model results in approximate solutions. Thus, we later evaluate the performance of the BM with respect to the GM model in terms of computational time and optimality of solutions. Finally, we compare run times for the MOGM and the BM.

Consider a typical scenario with a cash management system like the one depicted in Figure 2 with balances restricted to positive values $\left(b_{\min }=0\right.$ for accounts 1 and 2). Temporary idle cash balances can be invested in short-term marketable securities and bonds through an investment account 2 with an average return of $7.2 \%$ per annum ( $h=0.02 \%$ per day). Inflows and outflows to/from account 1 are charged with fixed costs $\gamma_{0}^{+}=\gamma_{0}^{-}=20 €$ and variable costs $\gamma_{1}^{+}=\gamma_{1}^{-}=0.01 \%$ defining a cost structure $\beta=\left\{\gamma_{0}^{+}, \gamma_{0}^{-}, \gamma_{1}^{+}, \gamma_{1}^{-}, h\right\}$ according to current bank practices in Spain. As normalizing factors for multiobjective optimization purposes, we set $C_{\max }$ and $R_{\max }$ to the respective cost and risk for a trivial policy that we next formally define.

Definition 1. A policy $x_{t}$ is called trivial when it takes no control action and the cash balance evolves freely. Mathematically, it can be expressed as:

$$
x_{t}=0
$$




$$
\begin{gathered}
b_{t}=b_{t}+f_{t} \\
\Gamma\left(x_{t}\right)=0 \\
c\left(x_{t}\right)=H\left(b_{t}\right) \\
1 \leq t \leq T, t \in \mathbb{N}, T \in \mathbb{N} .
\end{gathered}
$$

The use of a trivial policy as a benchmark allows us to straightforwardly compare the performance of policies because objective function values above one indicate a poorer performance than a trivial strategy. Hence, we set $C_{\max }=$ $4640.0 €$ and $R_{\max }=387.8 €$ and we assume that we know with certainty that cash flow for the next five working days is $\boldsymbol{f}=[1,1,4,-1,-3]$, figures in millions of Euros. After setting an arbitrary initial cash balance $b_{0}=20$, we are in a position to derive alternative policies using the MOGM, the BM and the GM. Considering a neutral cash manager $\left(w_{1}, w_{2}=0.5\right)$, the optimal solution to the MOGM encoded from equation (26) to (39) is given by the following sequence of control actions:

$$
\boldsymbol{x}_{1}=[0,6.1,0,1.3,2.4], \quad \boldsymbol{x}_{2}=[21.0,0,1.9,0,0] .
$$

The objective function value for this solution is 0.2249 , which is the combined cost and risk of the policy with respect to the trivial policy characterized by $C_{\max }$ and $R_{\max }$. We can also solve the BM encoded from equation (40) to (48), obtaining exactly the same solution described in (60). Run times to solve both the MOGM and the BM are below 0.01 seconds.

On the other hand, Gormley and Meade (2007) proposed the use of genetic algorithms to find sufficiently good solutions since approximate algorithms do not guarantee optimality. In their experiments, the authors maintained a population of 200 solutions for 30,000 generations in which parents are selected by binary tournament and with probability of mutation 0.4. For reproducibility purposes, we here replicate their implementation. More precisely, we implemented in Python a continuous genetic algorithm with single point crossover and generational replacement (Chelouah and Siarry, 2000). Following the recommendations in Gormley and Meade (2007), we generated individuals as follows:

- Sample $D$ from $(m, M)$ where $m(M)$ is a very small (large) number.

- Sample $V-D$ from $(0, M)$.

- Sample $\alpha_{1}$ from $(0,1)$ and set $d=D+\alpha_{1}(V-D)$.

- Sample $\alpha_{2}$ from $(0,1)$ and set $v=V-\alpha_{2}(V-d)$.

Although Gormley and Meade (2007) set $m=-\infty$ and $M=\infty$, in this example we set $m=0$ and $M=40$ to ensure a faster convergence of the genetic algorithm. Following with our numerical example, we now aim to minimize objective function (7) subject to GM policies using genetic algorithms. Since a genetic algorithm is an iterative process, we control iterations through the number of generations that evolve from the initial population. The larger the number of generations the deeper the search for better solutions. However, the larger the number of generations the longer the time required in a regular personal computer to obtain a solution as detailed in Table 1. 
Table 1: Performance of the genetic algorithm in the example.

\begin{tabular}{rrrr}
\hline Generations & Time $(\mathrm{sec})$ & Objective $\left(\mathcal{L}_{1}\right)$ & Optimality loss \\
\hline 100 & 9 & 0,5751 & $155,7 \%$ \\
1000 & 94 & 0,4514 & $100,7 \%$ \\
5000 & 485 & 0,3476 & $54,6 \%$ \\
10000 & 969 & 0,2251 & $0,1 \%$ \\
\hline
\end{tabular}

Note that the GM objective function value for 10,000 generations is very close to the values obtained by the MOGM and the BM. This observation can be made because in this case we already know the optimal value provided by our MOGM/BM. Otherwise, genetic algorithms are not able to distinguish between a local and a global minimum, hence complicating the selection of the number of generations or any other point to stop the search. In addition, run times for the GM using genetic algorithms drastically grow with the number of generations. Thus, a trade-off between computational time and solution quality is required. We solve this problem by using mathematical programming to solve the BM encoded from equation (41) to (48).

As an experimental validation, we report in Table 2 performance results comparing our BM using quadratic programming and the GM using genetic algorithms. With respect to empirical settings, we consider:

- a cost context $\beta=\{20 €, 20 €, 0.01 \%, 0.01 \%, 0.02 \%\}$ as described above;

- a Gaussian cash flow process with mean 0.1 and standard deviation 1, figures in millions of Euros;

- a Gaussian forecasting error with mean 0 and standard deviation 0.5;

- a minimum cash balance $b_{\min }=1.5$, equivalent to three standard deviations of the error distribution;

- a planning horizon of five days.

After setting an arbitrary initial cash balance $20 \%$ above $b_{\text {min }}$, we solve three batches (100 replicates each) of the problem using the BM and the GM model. In the case of GM model, we consider a genetic algorithm with increasing number of generations until a variation below 0.01 in the objective function between consecutive generations is obtained. In addition, we set the following parameters:

- a population of 200 individuals;

- a probability of mutation of 0.4 ;

- a lower bound $m=1.5$, equivalent to the minimum balance $b_{m i n}$;

- a higher bound $M=3 \cdot m=4.5$.

The average results shown in Table 2, where column Id identifies each of the experiments, show that run times for the BM are negligible with respect to the GM model and several orders of magnitude faster. In addition, we find a remarkable optimality loss for the GM with respect to the BM. 
Table 2: Performance of the BM and the GM for three experiments.

\begin{tabular}{crrrrrr}
\hline Id & \multicolumn{2}{c}{ Boundless Model (BM) } & \multicolumn{3}{c}{ Gormley-Meade model $(\mathrm{GM})$} & Optimality \\
\cline { 2 - 5 } & Time(sec) & Objective $\left(\mathcal{L}_{1}\right)$ & Gen. & Time $(\mathrm{sec})$ & Objective $\left(\mathcal{L}_{1}\right)$ & loss \\
\hline 1 & 0,005 & 0,37478 & 100 & 9 & 0,49727 & $32.7 \%$ \\
2 & 0,005 & 0,40643 & 500 & 43 & 0,44046 & $8,4 \%$ \\
3 & 0,005 & 0,40646 & 1,000 & 89 & 0,44731 & $10,1 \%$ \\
\hline
\end{tabular}

It is clear that computational time is an advantage of the BM with respect to the GM. However, one may wonder what is the required time to solve the $\mathrm{BM}$ and the MOGM, since both models rely on mathematical programming to solve the cash management problem. In what follows, we perform a comparison exercise to empirically confirm that the necessary run time to solve the BM is lower than that required to solve the MOGM.

As in the previous empirical exercise, we sample $T$ elements from the cash flow process $\mathcal{N}(0.1,1)$ that are used as a forecast input to both the MOGM and BM model. Next, we set an arbitrary initial cash balance $20 \%$ above $b_{\text {min }}$ and we generate 100 different forecast samples of variable length $T$. These samples, the initial cash balance, the minimum cash balance and the cost structure $\beta$ are enough to obtain the optimal policy by solving the MOGM model encoded from equation (26) to (39) and the BM model encoded from equation (40) to (48). Then, we implement both models using the Gurobi modeling framework (Gurobi Optimization, Inc, 2017) for Python and the run time results of 100 replicates for planning horizons ranging in $[5,20]$ in steps of five days are shown in Figure 3. Average run times for our BM are $38 \%$ lower with standard deviations $41 \%$ smaller than the MOGM confirming that a simpler formulation leads to remarkably lower run times to solve the CMP.

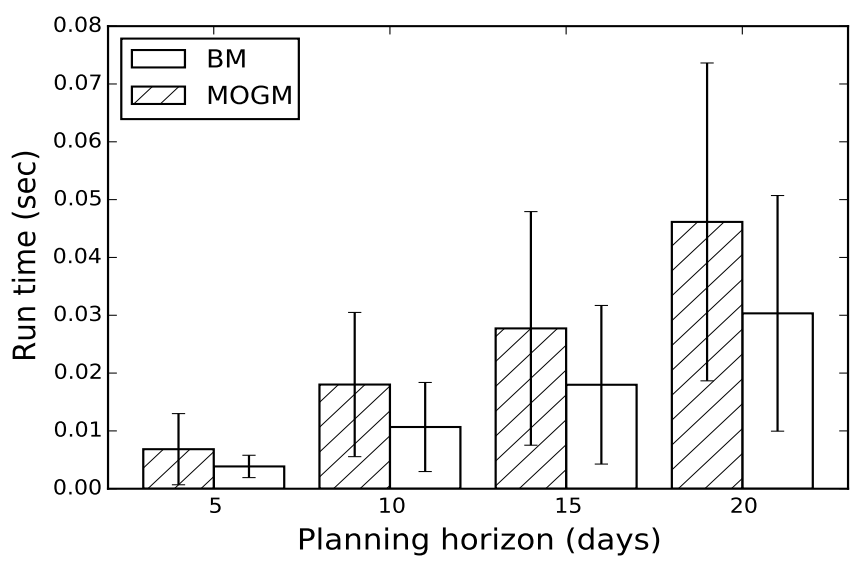

Figure 3: Run time for different planning horizons. 


\section{Sensitivity analysis to forecasting errors}

The critical step in the deployment of a BM is the procurement of forecasts. However, forecasts are characterized by some prediction error that necessarily impacts the performance of any model using forecasts as a key input. The main goal of this section is to perform a sensitivity analysis of the goodness of the solutions provided by our BM to changes in forecasting accuracy. We first describe our assumptions and our experimental methodology, and we finally discuss the results obtained.

\subsection{Assumptions}

We experiment on 54 real cash flow data sets of variable length, containing from 170 to 1508 observations. As a statistical summary, minimum and maximum values, means and standard deviations, and number of cash flow observations available for each company are shown in Table 3. The data set contains daily cash flows from 54 different small and medium companies in Spain with annual revenues of up to 10 million euro each, covering a date range of about 8 years. For illustrative purposes, we consider the following representative cost structure adjusted to current bank practices in Spain and very similar to those used in da Costa Moraes and Nagano (2014):

$$
\beta=\left\{\gamma_{0}^{+}=20 €, \gamma_{0}^{-}=20 €, \gamma_{1}^{+}=0.01 \%, \gamma_{1}^{-}=0.01 \%, h=0.02 \%\right\}
$$

We set a minimum balance $b_{\text {min }}$ equivalent to three standard deviations of the empirical error distribution for a planning horizon of five days to ensure a $99 \%$ of feasibility. Finally, we follow a multiobjective approach in which expected cost is defined as the average daily cost as in equation (5) and expected risk is defined as the standard deviation of daily cost as in equation (6). Then, under a compromise programming framework, the ultimate goal is to find the policy that minimizes equation (7).

\subsection{Methodology}

In order to evaluate the impact of the forecasting error in actual cost-risk performance, we propose Algorithm 1. From a cash flow data set stored in vector $\boldsymbol{f}$ we compute its standard deviation $\sigma_{f}$. Following the method described in SalasMolina et al. (2017), we obtain forecasts of controllable accuracy by assuming error $e_{t} \sim \mathcal{N}\left(0, \sigma_{e}\right)$ with $\sigma_{e}=p \cdot \sigma_{f}$ and with error proportion $p \in[0.001,1]$. Sample draws for different values of $p$ result in forecasts ranging from perfect prediction $\left(\sigma_{e} \approx 0\right)$ to an always-zero prediction $\sigma_{e}=\sigma_{f}$. Then, by computing the actual loss of policy $X$, namely $\mathcal{L}_{1}(X)$, when the actual cash balances are not $\hat{b}_{t}$ but $b_{t}=\hat{b}_{t}+e_{t}$, we can evaluate the impact of the forecasting error in the actual cost-risk performance of the model.

As in the comparison example described in Section 5.3, we set an initial cash balance $b_{0}$ to a value $20 \%$ above $b_{\min }$ as a feasible but non-conservative initial condition. Since we compare our BM to a trivial policy with the same initial condition, this setting does not interfere in the conclusions derived from the experiment. Then, given cost structure described in equation (61), we generated 100 replicates with different forecast samples of length five, equivalent to more than two years of total planning horizon for each of the 54 companies. Since we 
Table 3: Data sets description. Figures in thousands of $€$.

\begin{tabular}{|c|c|c|c|c|c|}
\hline Company & Min & Max & Mean & Std Dev & Length \\
\hline 1 & $-90,66$ & 902,69 & 0,22 & 39,71 & 622 \\
\hline 2 & $-565,06$ & 626,55 & 3,09 & 65,35 & 544 \\
\hline 3 & $-6.631,47$ & $6.710,41$ & $-7,44$ & 414,08 & 935 \\
\hline 4 & $-2.233,81$ & 727,63 & $-0,88$ & 170,28 & 893 \\
\hline 5 & $-182,62$ & 164,20 & 0,01 & 18,41 & 709 \\
\hline 6 & $-689,70$ & 562,69 & $-0,41$ & 72,83 & 688 \\
\hline 7 & $-300,09$ & 829,05 & 0,26 & 65,52 & 555 \\
\hline 8 & $-242,06$ & 113,14 & $-0,14$ & 31,89 & 789 \\
\hline 9 & $-4.703,91$ & $4.733,65$ & $-3,10$ & 658,57 & 754 \\
\hline 10 & $-1.115,80$ & 787,24 & $-1,89$ & 83,55 & 788 \\
\hline 11 & $-1.915,34$ & 307,44 & 1,33 & 107,38 & 428 \\
\hline 12 & $-615,77$ & $7.713,80$ & $-0,11$ & 338,26 & 555 \\
\hline 13 & $-1.183,62$ & $2.274,46$ & $-0,39$ & 287,26 & 549 \\
\hline 14 & $-769,28$ & 927,11 & $-1,00$ & 142,23 & 606 \\
\hline 15 & $-551,11$ & 556,13 & 0,39 & 114,95 & 696 \\
\hline 16 & $-220,49$ & 226,11 & $-0,50$ & 18,25 & 577 \\
\hline 17 & $-2.253,22$ & $2.501,26$ & 0,63 & 175,38 & 991 \\
\hline 18 & $-287,58$ & 263,61 & $-0,09$ & 26,41 & 610 \\
\hline 19 & $-161,73$ & 154,82 & $-3,08$ & 25,47 & 640 \\
\hline 20 & $-150,00$ & 160,38 & $-0,37$ & 15,40 & 632 \\
\hline 21 & $-700,00$ & 531,66 & $-0,54$ & 65,06 & 730 \\
\hline 22 & $-2.442,94$ & $1.388,74$ & $-2,15$ & 280,20 & 509 \\
\hline 23 & $-2.898,68$ & $2.898,68$ & $-2,54$ & 336,42 & 586 \\
\hline 24 & $-3.025,05$ & $3.178,51$ & $-4,05$ & 247,62 & 1285 \\
\hline 25 & $-1.969,42$ & $2.011,31$ & $\begin{array}{r}-0,39 \\
-1,30\end{array}$ & 174,53 & 600 \\
\hline 26 & $-107,28$ & 155,63 & $-0,05$ & 18,64 & 708 \\
\hline 27 & $-70,99$ & 118,38 & 2,75 & 16,87 & 340 \\
\hline 28 & $-324,81$ & 390,08 & $-0,79$ & 48,56 & 901 \\
\hline 29 & $-900,41$ & 558,88 & $-0,34$ & 65,59 & 574 \\
\hline 30 & $-188,79$ & 198,15 & $-0,46$ & 17,59 & 536 \\
\hline 31 & $-1.344,75$ & 349,45 & $-2,75$ & 119,68 & 336 \\
\hline 32 & $-359,16$ & 245,04 & 2,71 & 48,77 & 860 \\
\hline 33 & $-943,25$ & 955,89 & $-1,18$ & 78,27 & 670 \\
\hline 34 & $-1.149,40$ & 496,55 & $-1,39$ & 108,36 & 1490 \\
\hline 35 & $-410,71$ & 291,91 & $-0,55$ & 57,86 & 600 \\
\hline 36 & $-78,72$ & 118,40 & 4,45 & 18,64 & 357 \\
\hline 37 & $-2.288,85$ & $2.184,18$ & $-10,16$ & 180,89 & 497 \\
\hline 38 & $-619,33$ & 196,64 & $-11,18$ & 67,60 & 193 \\
\hline 39 & $-64,71$ & 65,67 & $-0,11$ & 11,66 & 829 \\
\hline 40 & $-256,27$ & 369,14 & 0,24 & 103,05 & 291 \\
\hline 41 & $-626,65$ & 643,39 & $-5,55$ & 96,41 & 300 \\
\hline 42 & $-370,21$ & 368,46 & 0,47 & 23,11 & 749 \\
\hline 43 & $-658,44$ & 733,95 & $-0,37$ & 131,40 & 832 \\
\hline 44 & $-1.187,40$ & $1.203,41$ & $-1,83$ & 115,28 & 378 \\
\hline 45 & $-1.071,96$ & $1.128,00$ & 0,58 & 127,81 & 881 \\
\hline 46 & $-511,63$ & 738,32 & 10,06 & 75,56 & 411 \\
\hline 47 & $-10.374,88$ & $4.782,62$ & $\begin{array}{r}10,90 \\
-22,94\end{array}$ & 723,62 & 532 \\
\hline 48 & $-2.070,38$ & $2.030,93$ & $-5,58$ & 255,32 & 581 \\
\hline 49 & $-107,84$ & 127,25 & $-2,07$ & 19,96 & 573 \\
\hline 50 & $-2.625,18$ & $2.219,57$ & $-2,45$ & 351,19 & 374 \\
\hline 51 & $-4.198,83$ & $4.816,62$ & 151,28 & 970,81 & 1222 \\
\hline 52 & $-3.254,65$ & $7.006,59$ & 89,72 & 494,93 & 1220 \\
\hline 53 & $-1.968,77$ & 384,84 & 7,76 & 117,51 & 738 \\
\hline 54 & $-10.213,56$ & $15.321,00$ & 9,61 & $1.124,10$ & 589 \\
\hline
\end{tabular}

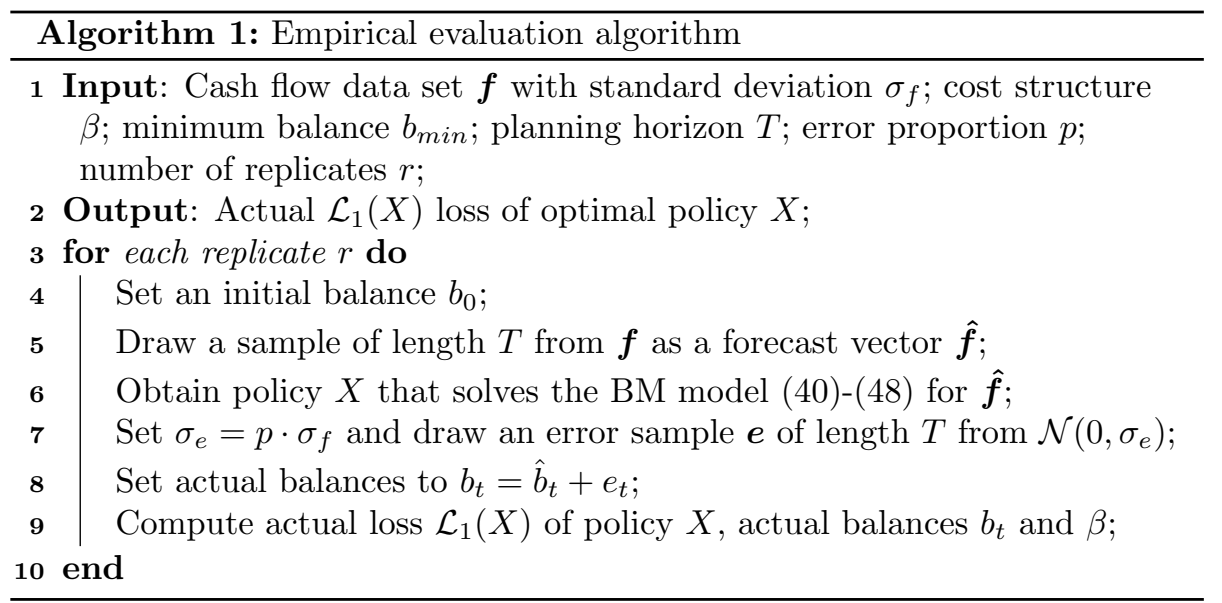


consider eleven error proportions, from an almost perfect cash flow prediction to an unpredictable cash flow, we cover a whole range of possible situations. As a result, we perform 59,400 experiments. Note also that, for comparative purposes, we consider that every policy is fixed, i.e., it cannot be modified during the planning horizon. In practice, the optimization process would be repeated either when new information about actual cash flows or when some error tolerances are exceeded.

\subsection{Results and discussion}

The results from the evaluation for all 54 companies grouped by error proportion $(p)$ are shown in Figure 4 by means of a box-plot. Horizontal bands inside the box show median values, the bottom and the top of the box represent the first and third quartile, and whiskers the 5th and 95th percentiles. The results show that actual cost-risk losses derived from the BM policy in comparison to a trivial policy are minimum when near-to-perfect predictions are possible (error proportion is 0.001). However, remarkable savings can be achieved for higher but reasonable error proportions. In practice, one can consider that error proportions below 0.5 are achievable since a high percentage of cash flows are usually known with certainty in the short-term due to payment agreements with customers, suppliers, banks and employees. This point is particularly common for small and medium size companies such as those included in our data set from Table 3. Indeed, the assumption of perfect predictions is common in previous cash management works such as Baumol (1952), Golden et al. (1979) and de Avila Pacheco and Morabito (2011).

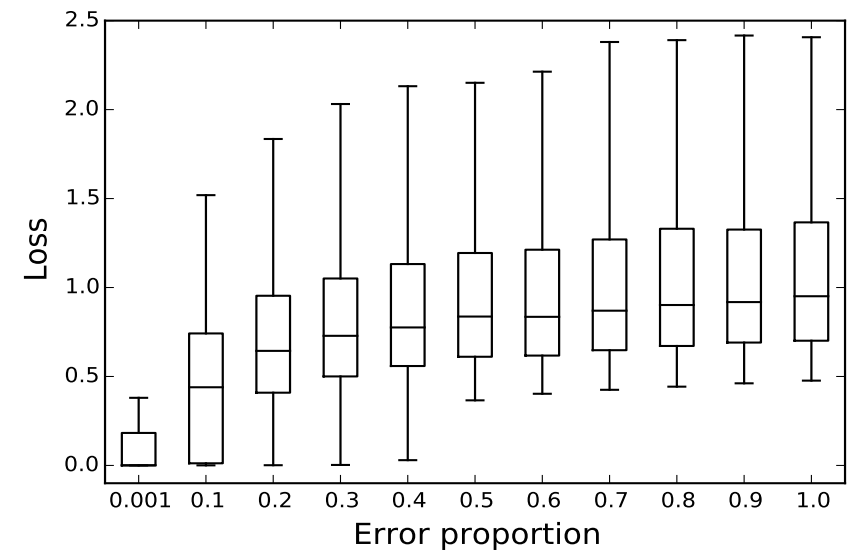

Figure 4: Median cost-risk loss of the BM for different forecasting errors.

The results from Figure 4 show a high variability in cost-risk losses even for low error proportions. In the long term, our BM proves to achieve remarkable savings with respect to the benchmark, but at the same time, potential losses can occur. This effect is mainly caused by our experimental setting with a five days planning horizon and fixed policy that we use to evaluate the impact of forecasting horizons. In practice, cash managers can obtain a new policy at each time step to replace the previous one when new current balances are available 
as described in Bemporad and Morari (1999) and Camacho and Alba (2013), hence providing the desired feedback control.

Another possible reason for this high variability is the underlying cash flow process faced by each company in our data set. Since a value above one implies a poorer performance than the benchmark, we report in Table 4 detailed results for the number of companies below this threshold value for different prediction error proportions at different quantiles. We aim to study the expectation for a given company to achieve better results than the benchmark. For instance, we expect that $61 \%$ of companies with error proportion 0.2 obtain better results than the benchmark $75 \%$ of the times. Note that line $Q_{50}$ in Table 4 would correspond to the horizontal line within the box as in Figure 4 for a particular company, line $Q_{75}$ to the upper box limit, and line $Q_{95}$ is the upper whisker. From these results, we conclude that companies can differently benefit from deploying a $\mathrm{BM}$, even in the case of a fixed policy for the whole planning horizon in our experimental setting. By implementing the method proposed in Algorithm 1, cash managers are able to determine the expected cost-risk loss of deploying a $\mathrm{BM}$ under a particular cash flow process.

Table 4: Percentage of companies below loss threshold one for different prediction errors and quantiles.

\begin{tabular}{lrrrrrrrrrrr}
\hline Proportion & 0,001 & 0,1 & 0,2 & 0,3 & 0,4 & 0,5 & 0,6 & 0,7 & 0,8 & 0,9 & 1.0 \\
\hline$Q_{50}$ & 100 & 98 & 98 & 93 & 96 & 93 & 94 & 81 & 83 & 83 & 67 \\
$Q_{75}$ & 100 & 87 & 61 & 43 & 24 & 13 & 9 & 7 & 2 & 0 & 0 \\
$Q_{95}$ & 98 & 15 & 4 & 0 & 0 & 0 & 0 & 0 & 0 & 0 & 0 \\
\hline
\end{tabular}

It is also worth noting that efforts in improving forecasting accuracy are highly rewarded using the BM. For example, consider a cash manager that is able to achieve forecasting errors of, at most, $40 \%$ of the standard deviation of past cash flows $(p=0.4)$, which can be considered a reasonable target. By using predictions and our BM, we observe in Figure 4 that more than $20 \%$ of the total loss can be saved. Going one step further, if the cost of obtaining predictions is less than that $20 \%$, our hypothetical cash manager should deploy a BM. Summarizing, our BM proves to be significantly robust to forecasting errors since remarkable cost-risk savings with respect to a trivial policy can be obtained even for almost purely random cash flows. This fact must encourage cash managers to produce better cash flow forecasts since improvements in accuracy are highly rewarded.

\section{Conclusions}

Deriving cash management policies is by no means straightforward. In this paper, we provide cash managers with a modelling framework to deal with cash flow uncertainty. We also solve one serious limitation of cash management models based on bounds: its difficulty to obtain optimal solutions. We here propose to linearize the cash management problem in an attempt to provide a sound mathematical programming framework that returns optimal policies. Once the cash management problem is formulated as a linear program with both continuous and auxiliary binary variables, the bounds that determines the 
policy of a BBM can be expressed as a set of additional constraints. We illustrate this approach by providing a multiobjective reformulation of the Gormley and Meade (2007) model within a compromise programming framework where cost and risk (but possibly other) are goals to optimize.

Furthermore, we propose a new BM for multiobjective cash management which departs from the state-of-the-art bound-based approach in the fact that is not constrained by any particular form of policy. Since the ultimate goal of the cash management problem is to find the best policy instead of a set of bounds that determines the policy, we show that the constraints imposed by BBMs are redundant and can be removed from the optimization problem.

Since forecasts are characterized by some prediction error, we also analyze the impact of predictive accuracy in cost-risk performance of policies derived from our BM showing that it is highly robust to forecasting errors. Note also that the methodology used to analyze the impact of forecasting errors also supports decision-making by estimating the reward that can be obtained through the use of better forecasts. Deployment costs can then be compared to estimated savings to decide if further effort in improving predictive accuracy is worthwhile.

Further research may consider multiple bank accounts and different cost functions in an attempt to close the gap between theory and practice. In practice, cash transactions between multiple bank accounts with different cash flow characteristics are common in most companies. In order to make the BM more immune to unexpected losses due to unreliable forecasts, robust optimization (Ben-Tal et al., 2009) represents a suitable future line of work. Finally, more complex cost functions, such as time dependent costs or piece-wise cost functions, can also be included in the analysis in order to achieve a better representation of the cash management problem.

\section{Acknowledgments}

Work partially funded by projects Collectiveware TIN2015-66863-C2-1-R (MINECO/ FEDER) and 2014 SGR 118.

\section{References}

Artzner, P., Delbaen, F., Eber, J.-M., and Heath, D. (1999). Coherent measures of risk. Mathematical finance, 9(3):203-228.

Baccarin, S. (2009). Optimal impulse control for a multidimensional cash management system with generalized cost functions. European Journal of Operational Research, 196(1):198-206.

Ballestero, E. and Romero, C. (1998). Multiple criteria decision making and its applications to economic problems. Springer Science \& Business Media.

Bar-Ilan, A., Perry, D., and Stadje, W. (2004). A generalized impulse control model of cash management. Journal of Economic Dynamics and Control, 28(6):1013-1033.

Baumol, W. J. (1952). The transactions demand for cash: An inventory theoretic approach. The Quarterly Journal of Economics, 66(4):545-556. 
Bazaraa, M. S., Jarvis, J. J., and Sherali, H. D. (2011). Linear programming and network flows. John Wiley \& Sons.

Bemporad, A. and Morari, M. (1999). Control of systems integrating logic, dynamics, and constraints. Automatica, 35(3):407-427.

Ben-Tal, A., El Ghaoui, L., and Nemirovski, A. (2009). Robust optimization. Princeton University Press.

Branke, J., Deb, K., and Miettinen, K. (2008). Multiobjective optimization: Interactive and evolutionary approaches. Springer Science \& Business Media.

Camacho, E. F. and Alba, C. B. (2013). Model predictive control. Springer Science \& Business Media.

Chelouah, R. and Siarry, P. (2000). A continuous genetic algorithm designed for the global optimization of multimodal functions. Journal of Heuristics, 6(2):191-213.

Chen, X. and Simchi-Levi, D. (2009). A new approach for the stochastic cash balance problem with fixed costs. Probability in the Engineering and Informational Sciences, 23(04):545-562.

Constantinides, G. M. and Richard, S. F. (1978). Existence of optimal simple policies for discounted-cost inventory and cash management in continuous time. Operations Research, 26(4):620-636.

da Costa Moraes, M. B. and Nagano, M. S. (2014). Evolutionary models in cash management policies with multiple assets. Economic Modelling, 39:1-7.

da Costa Moraes, M. B., Nagano, M. S., and Sobreiro, V. A. (2015). Stochastic cash flow management models: A literature review since the 1980s. In Decision Models in Engineering and Management, pages 11-28. Springer.

de Avila Pacheco, J. V. and Morabito, R. (2011). Application of network flow models for the cash management of an agribusiness company. Computers 86 Industrial Engineering, 61(3):848-857.

Girgis, N. M. (1968). Optimal cash balance levels. Management Science, 15(3):130-140.

Golden, B., Liberatore, M., and Lieberman, C. (1979). Models and solution techniques for cash flow management. Computers $\&$ Operations Research, $6(1): 13-20$.

Gormley, F. M. and Meade, N. (2007). The utility of cash flow forecasts in the management of corporate cash balances. European Journal of Operational Research, 182(2):923-935.

Gregory, G. (1976). Cash flow models: a review. Omega, 4(6):643-656.

Gurobi Optimization, Inc (2017). Gurobi optimizer reference manual.

Keown, A. J. and Martin, J. D. (1977). A chance constrained goal programming model for working capital management. The Engineering Economist, $22(3): 153-174$. 
Miller, M. H. and Orr, D. (1966). A model of the demand for money by firms. The Quarterly Journal of Economics, 80(3):413-435.

Neave, E. H. (1970). The stochastic cash balance problem with fixed costs for increases and decreases. Management Science, 16(7):472-490.

Nocedal, J. and Wright, S. J. (2006). Numerical optimization 2nd. Springer.

Park, C. S. and Herath, H. S. (2000). Exploiting uncertainty —investment opportunities as real options: a new way of thinking in engineering economics. The Engineering Economist, 45(1):1-36.

Penttinen, M. J. (1991). Myopic and stationary solutions for stochastic cash balance problems. European Journal of Operational Research, 52(2):155-166.

Rockafellar, R. T. and Uryasev, S. (2002). Conditional value-at-risk for general loss distributions. Journal of banking \&3 finance, 26(7):1443-1471.

Salas-Molina, F., Martin, F. J., Rodríguez-Aguilar, J. A., Serrà, J., and Arcos, J. L. (2017). Empowering cash managers to achieve cost savings by improving predictive accuracy. International Journal of Forecasting, 33(2):403-415.

Salas-Molina, F., Pla-Santamaria, D., and Rodriguez-Aguilar, J. A. (2016). A multi-objective approach to the cash management problem. Annals of Operations Research, pages 1-15.

Srinivasan, V. and Kim, Y. H. (1986). Deterministic cash flow management: state of the art and research directions. Omega, 14(2):145-166.

Stone, B. K. (1972). The use of forecasts and smoothing in control-limit models for cash management. Financial Management, 1(1):72-84.

Stone, B. K. and Miller, T. W. (1987). Daily cash forecasting with multiplicative models of cash flow patterns. Financial Management, 16(4):45-54.

Xu, X. and Birge, J. R. (2008). Operational decisions, capital structure, and managerial compensation: A news vendor perspective. The Engineering Economist, 53(3):173-196.

Yu, P.-L. (1985). Multiple criteria decision making: concepts, techniques and extensions. Plenum Press. 